\title{
Rifampin-associated tubulointersititial nephritis and Fanconi syndrome presenting as hypokalemic paralysis
}

\author{
Hong Ki Min ${ }^{1}$, Eun Oh Kim', Sang Ju Lee ${ }^{1}$, Yoon Kyung Chang ${ }^{1}$, Kwang Sun Suh², Chul Woo Yang ${ }^{1}$,
} Suk Young Kim ${ }^{1}$ and Hyeon Seok Hwang ${ }^{1 *}$

\begin{abstract}
Background: Rifampin is one of the most important drugs in first-line therapies for tuberculosis. The renal toxicity of rifampin has been reported sporadically and acute tubulointerstitial nephritis (ATIN) is a frequent histological finding. We describe for the first time a case of ATIN and Fanconi syndrome presenting as hypokalemic paralysis, associated with the use of rifampin.

Case presentation: A 42-year-old man was admitted with sudden-onset lower extremity paralysis and mild renal insufficiency. He had been treated for pulmonary tuberculosis with isoniazid, rifampin, and ethambutol for 2 months. Laboratory tests revealed proteinuria, profound hypokalemia, hyperchloremic metabolic acidosis with a normal anion gap, positive urine anion gap, hypophosphatemia with hyperphosphaturia, hypouricemia with hyperuricosuria, glycosuria with normal serum glucose level, generalized aminoaciduria, and $\beta 2$-microglobulinuria. A kidney biopsy revealed findings typical of ATIN and focal granular deposits of immunoglubulin A and complement 3 in the glomeruli and tubules. Electron microscopy showed epithelial foot process effacement and electron-dense deposits in the subendothelial and mesangial spaces. Cessation of rifampin resolved the patient's clinical presentation of Fanconi syndrome, and improved his renal function and proteinuria.

Conclusion: This case demonstrates that rifampin therapy can be associated with Fanconi syndrome presenting as hypokalemic paralysis, which is a manifestation of ATIN. Kidney function and the markers of proximal tubular injury should be carefully monitored in patients receiving rifampin.
\end{abstract}

Keywords: Rifampin, Fanconi syndrome, Tubulointerstitial nephritis, Hypokalemic paralysis

\section{Background}

Rifampin is one of the most effective antibiotics used for the treatment of tuberculosis. The renal toxicity of rifampin has been reported sporadically and acute renal failure is a frequent clinical presentation [1]. Histologically, rifampin nephrotoxicity is associated with acute tubulointerstitial nephritis (ATIN), tubular necrosis, papillary necrosis, acute cortical necrosis, and minimal change disease [1-3]. Of these, ATIN and tubular necrosis are the most common and frequently develop upon reintroduction of the drug or during intermittent therapy.

\footnotetext{
* Correspondence: hwanghs@catholic.ac.kr

${ }^{1}$ Division of Nephrology, Department of Internal Medicine, The Catholic University of Korea, Seoul, Korea

Full list of author information is available at the end of the article
}

Acquired Fanconi syndrome occurs at any age as an autoimmune disorder or after exposure to noxious agents $[4,5]$. It is characterized by a generalized transport defect in the proximal tubules and can be associated with drug-induced ATIN [6-8]. However, neither Fanconi syndrome nor associated hypokalemic paralysis has ever been reported in patients with rifampinassociated ATIN. We describe a case of ATIN and Fanconi syndrome presenting as hypokalemic paralysis in a patient receiving rifampin.

\section{Case presentation}

A 42-year-old man was hospitalized with sudden-onset weakness in both lower extremities. The patient had been treated with isoniazid $300 \mathrm{mg}$, rifampin $450 \mathrm{mg}$, 
and ethambutol $800 \mathrm{mg}$ daily for pulmonary tuberculosis during the past two months. He had no history of exposure to antituberculosis antibiotics before beginning therapy. His compliance to antibiotics was excellent after the treatment for pulmonary tuberculosis and he denied the interruption on these regimens. He reported no constitutional symptoms, history of hyperthyroidism or paralysis.

A physical examination indicated that the muscle strength of the lower extremities was $2 / 5$ of normal. The rest of the physical examination was unremarkable. A chest radiograph revealed reticular opacities on the bilateral upper lung fields, consistent with pulmonary tuberculosis. His laboratory data included hemoglobin $13.0 \mathrm{mg} / \mathrm{dL}$, leukocyte count $9900 / \mu \mathrm{L}$, and platelet count $290,000 / \mu \mathrm{L}$. His biochemical data for serum and urine on admission are shown in Table 1. Profound hypokalemia, hypophosphatemia, and hypouricemia were present. Serum pH, bicarbonate level and anion gap indicated hyperchloremic metabolic acidosis with normal anion gap. The urinary anion gap was positive $(10.2 \mathrm{mEq} / \mathrm{L})$, suggesting the presence of renal tubular acidosis. Urinalysis showed normoglycemic glucosuria, $\beta_{2}$-microglobulinuria, $\mathrm{pH} 5.5,2.5 \mathrm{~g} /$ day proteinuria and microscopic hematuria. The fractional excretion of potassium was $29.3 \%$ (normal range, $4 \%-$ $16 \%$ ); the calculated ratio of the maximal tubular transport of phosphate reabsorption to the glomerular filtration rate (TmP/GFR) was $0.24 \mathrm{mg} / \mathrm{dL}$ (normal range, $2.3-4.3 \mathrm{mg} /$ $\mathrm{dL})$; and the fractional excretion of uric acid was $77.4 \%$ (normal range, 6\%-20\%). Generalized hyperaminoaciduria was detected with liquid chromatography-tandem mass spectrometry. These findings suggest generalized proximal tubular dysfunction with wasting of bicarbonate, glucose, protein, potassium, phosphate, and uric acid.

Additional blood tests were performed to determine other possible causes of the patient's hypokalemia. His plasma renin activity and serum level of aldosterone

Table 1 Biochemical Data on Admission and after Rifampin Withdrawal

\begin{tabular}{|c|c|c|c|c|c|}
\hline & Admission & 2 weeks & 3 months & 6 months & 8 months \\
\hline \multicolumn{6}{|l|}{ Serum } \\
\hline Urea nitrogen (mg/dL) & 13.9 & - & 18.5 & 15.9 & 15.9 \\
\hline Creatinine (mg/dL) & 1.4 & - & 1.12 & 1.18 & 1.09 \\
\hline Potassium (mmol/L) & 2.0 & 3.8 & 3.8 & 3.7 & 4.3 \\
\hline $\mathrm{pH}$ & 7.289 & 7.260 & 7.387 & 7.381 & 7.367 \\
\hline Bicarbonate (mmol/L) & 12.4 & 14.5 & 22.8 & 23.3 & 24.4 \\
\hline Phosphate (mg/dL) & 1.2 & 2.7 & 5.2 & 4.4 & 4.6 \\
\hline Uric acid (mg/dL) & 1.2 & 1.3 & 3.9 & 4.5 & 4.2 \\
\hline Sodium (mmol/L) & 141 & 140 & 140 & 140 & 142 \\
\hline Chloride (mmol/L) & 114 & 112 & 103 & 103 & 106 \\
\hline Magnesium (mg/dL) & 2.1 & 2.3 & 2.5 & 2.3 & 2.3 \\
\hline Albumin (g/dL) & 4.7 & - & - & 4.3 & 4.3 \\
\hline \multicolumn{6}{|l|}{ Urine } \\
\hline 24-h glucose (g/day) & 16.90 & - & 0.05 & 0.06 & - \\
\hline$\beta_{2}$-microglobulin $(\mu \mathrm{g} / \mathrm{L})^{*}$ & $>20000$ & - & 1238 & 265 & - \\
\hline Potassium (mEq/L) & 7.2 & & 29.5 & 61.4 & 67 \\
\hline Phosphate (mg/dL) & 11.7 & & 33.7 & 69.5 & 96.8 \\
\hline Uric acid (mg/dL) & 11.4 & & 28.1 & - & 67.3 \\
\hline Creatinine $(\mathrm{mg} / \mathrm{dL})$ & 17.2 & & 38.99 & 189.7 & 171 \\
\hline $\mathrm{FE}_{\mathrm{K}}(\%)^{\dagger}$ & 29.33 & - & 22.29 & 10.32 & 9.80 \\
\hline $\mathrm{TmP} / \mathrm{GFR}(\mathrm{mg} / \mathrm{dL})^{\ddagger}$ & 0.24 & - & 4.23 & 3.96 & 3.98 \\
\hline$F E_{\cup A}(\%)^{\S}$ & 77.41 & - & 20.69 & - & 10.2 \\
\hline $\mathrm{pH}$ & 5.5 & 6.0 & 5.0 & 5.0 & 5.5 \\
\hline 24-h protein (g/day) & 2.50 & 0.91 & 0.10 & 0.14 & 0.01 \\
\hline
\end{tabular}

Note: Replacement of potassium chloride and sodium bicarbonate was interrupted at 3 months after admission.

*Normal, less than $370 \mathrm{mg} / \mathrm{L}$.

+ Normal, 4-16\%.

${ }^{\ddagger}$ Normal, 2.3 to 4.3

${ }^{\S}$ Normal, 6-20\%.

$\mathrm{FE}_{\mathrm{K}}$, fractional excretion of potassium; TmP/GFR, tubular maximal transport of phosphate reabsorption to the glomerular filtration rate transport; $\mathrm{FE}_{\mathrm{UA}}$, fractional excretion of uric acid. 
were $21.6 \mathrm{ng} / \mathrm{mL} / \mathrm{h}$ and $30.5 \mathrm{ng} / \mathrm{dL}$, respectively. His thyroid-stimulating hormone and free thyroxine levels were within the normal ranges. Serum and urinary protein immunoelectrophoresis showed no evidence of monoclonal gammopathy, and immunological surveys of autoantibodies were negative. The patient's reticulocyte count, serum lactate dehydrogenase, and liver enzymes levels were within the normal ranges.

An ultrasonographic examination showed a normalsized kidney, with slightly increased echogenicity in the bilateral renal parenchyma. A renal biopsy showed extensive mononuclear cell infiltrates, including epithelioid histiocytes and eosinophils, mild interstitial fibrosis, and tubular atrophy (Figure 1A and B). Ziehl-Neelsen staining for acid-fast bacilli and PCR detection of Mycobacterium tuberculosis in the renal biopsy specimen were negative. Focal granular deposits of immunoglobulin A (IgA) and complement 3 (C3) were demonstrated in the tubules (Figure $1 \mathrm{C}$ and $\mathrm{D}$ ). The same immunofluorescent positivity was also shown in glomerular mesangium, and electron microscopy showed electrondense deposits in the subendothelial and mesangial
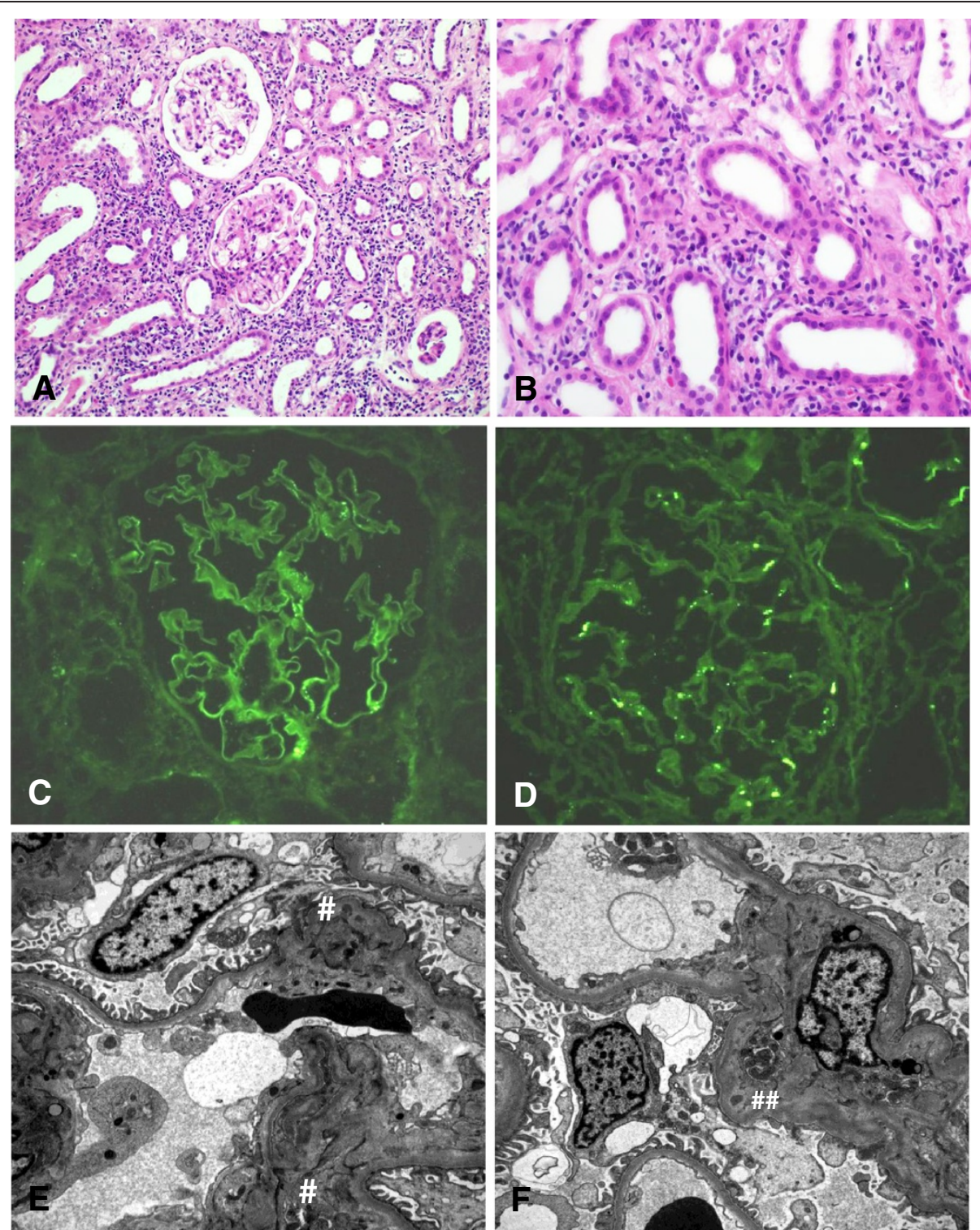

Figure 1 Pathologic findings in a patient with rifampin-associated acute tubulointerstitial nephritis and Fanconi syndrome. Light microscopy revealed the extensive mononuclear cell infiltrates including epithelioid histiocytes and eosinophils, mild interstitial fibrosis and tubular atrophy (A: original magnification X 100; B: original magnification x 400). Immunofluorescent stains showed focal granular deposits of immunoglobulin A (C) and complement 3 (D) in mesangial spaces and tubules. Subendothelial electron-dense deposits (E) and expanded mesangial spaces with electron dense deposits $(\mathbf{F})$ were visible using electron microscopy. 
spaces (Figure 1E and F). These findings were consistent with IgA nephropathy accompanied by focal immune deposits along the tubules. Half the epithelial foot processes were effaced, and there were no pathological findings in the mitochondria.

Potassium chloride supplementation was given to treat the patient's hypokalemic paralysis. His muscle strength increased one day after potassium chloride supplementation. We substituted rifampin with levofloxacin, but his other antituberculosis antibiotics remained the same. Supplementation with potassium chloride and sodium bicarbonate was continued, and the patient was discharged on hospital day 14. The biochemical markers associated with Fanconi syndrome were significantly resolved, and he experienced no paralytic symptoms after discharge. His proteinuria and microscopic hematuria was also improved, and we discontinued supplementation with potassium chloride and sodium bicarbonate at the three-month follow-up. The patient's renal function and proximal tubular function remained stable at the last follow-up.

\section{Discussion}

We have reported for the first time rifampin-associated ATIN and Fanconi syndrome presenting as hypokalemic paralysis. The typical findings of ATIN were present in a kidney biopsy specimen from the patient, and generalized proximal tubular dysfunction was demonstrated by his biochemical parameters. The symptoms of Fanconi syndrome disappeared after the discontinuation of rifampin, which strongly indicates that rifampin was the causative agent of Fanconi syndrome.

The clinical manifestations of rifampin-associated nephrotoxicity can be mainly classified into two groups, depending on the anti-rifampin antibody induced $[9,10]$. In patients treated with an interrupted regimen, the rifampin-dependent antibody produces acute tubular necrosis requiring dialysis, intravascular hemolysis, and flu-like symptoms. In contrast, the continuous administration of rifampin has been described as progressing more insidiously. In this setting, tests for anti-rifampin antibody are uniformly negative, and the degree of interstitial inflammation is much greater than that of acute tubular necrosis [11]. Our patient had no history of prior exposure to rifampin or interruption on regimen, and reported no constitutional symptoms. Furthermore, the results of a kidney biopsy were compatible with the latter condition. These findings suggest that ATIN occurred independently of anti-rifampin antibodies, and the absence of intravascular hemolysis further supports this view.

Several types of drugs cause ATIN-induced Fanconi syndrome, and mitochondrial dysfunction, direct tubular toxicity, and immune reactions have been considered to be the pathogenic mechanisms $[4,12,13]$. In this patient, a renal biopsy showed no structural abnormalities of the mitochondria, but demonstrated extensive lymphocytic infiltration with eosinophils. Immunofluorescent staining also showed focal deposits of IgA and C3 along the tubules. These findings suggest that hypersensitive immune-mediated injury to the tubular membrane was the pathogenic mechanism of Fanconi syndrome, and anti-tubular basement membrane antibodies have been suggested to induce ATIN in such cases [10-14].

The coexistence of glomerular and tubular lesions is relatively rare in drug-induced ATIN. In our patient, the same immunofluorescent positivity shown in the damaged tubules was demonstrated in the glomerular mesangium, and electron-dense deposits were also present in the subendothelial and mesangial spaces. These findings suggest a relationship between the tubular insult and the immune injury progressing to the glomeruli. We infer that the release of tubular antigens as a consequence of rifampininduced immunological injury gave rise to superimposed immune complex deposition in the glomeruli, which progressed further to IgA nephropathy [15-17].

Hypokalemia is usually mild to moderate in Fanconi syndrome [18]. However, profound hypokalemia with muscle paralysis was the presenting feature in this patient. The mechanisms of renal potassium wasting were considered to be as follows. First, the reduced reabsorption of potassium in the proximal tubule increased the urinary potassium loss. Second, the bicarbonaturia resulting from the impaired bicarbonate reabsorption in the proximal tubule enhanced the renal potassium excretion [19]. Third, the increased aldosterone level in our patient suggested that the reduced proximal sodium reabsorption increased the distal delivery of sodium, resulting in the depletion of the extracellular fluid volume and secondary hyperaldosteronism [20].

The management of drug-induced ATIN includes the immediate discontinuation of the offending agent and the use of corticosteroids [12,13]. A short course of corticosteroid is usually administered to patients with rifampininduced ATIN, and severe renal failure, prolonged renal failure, or crescentic nephritis increases the need for corticosteroids [21,22]. In our case, cessation of the offending agent without corticosteroid restored the patient's renal function and resolved his proteinuria. Furthermore, the clinical and biochemical presentations of Fanconi syndrome were completely reversed. Therefore, we suggest that the discontinuation of rifampin alone can improve the ATIN and Fanconi syndrome in such patients, when the patient shows insidious clinical progress without serious renal failure or crescentic nephritis.

\section{Conclusion}

In conclusion, this case illustrates that rifampin can be associated with ATIN and Fanconi syndrome, presenting as hypokalemic paralysis. Therefore, Fanconi syndrome 
should be considered in the spectrum of renal diseases associated with rifampin. Renal function, in particular proximal tubular function, should be closely monitored when rifampin is used.

\section{Consent}

Written informed consent was obtained from the patient for publication of this case report.

\begin{abstract}
Abbreviations
ATIN: Acute tubulointerstitial nephritis; C3: Complement 3; FE: Fractional excretion of potassium; FE UA: Fractional excretion of uric acid; IgA: Immunoglobulin A; TmP/GFR: Tubular maximal transport of phosphate reabsorption to the glomerular filtration rate transport.
\end{abstract}

\section{Competing interests}

The authors declare that they have no competing interests.

\section{Authors' contributions}

HKM, EOK, SJL, YKC, SYK, and HSH treated the patient and provided data about the history and laboratory results in this report. HSH performed the renal biopsy. KSS interpreted the kidney biopsy. The manuscript was prepared by HKM, CWY, and HSH. All authors participated in discussions about the manuscript and approved the final version.

\section{Acknowledgments}

This study was supported by the Basic Science Research Program through the National Research Foundation of Korea (NRF) funded by the Ministry of Education, Science and Technology (2012R1A1A1009690).

\section{Author details}

'Division of Nephrology, Department of Internal Medicine, The Catholic University of Korea, Seoul, Korea. ${ }^{2}$ Department of Pathology, Chungnam National University College of Medicine, Daejeon, Korea.

Received: 13 November 2012 Accepted: 14 January 2013

Published: 16 January 2013

\section{References}

1. Muthukumar T, Jayakumar M, Fernando EM, Muthusethupathi MA: Acute renal failure due to rifampicin: a study of 25 patients. Am J Kidney Dis 2002, 40(4):690-696.

2. Nessi R, Bonoldi GL, Redaelli B, di Fillippo G: Acute renal failure after rifampicin: a case report and survey of the literature. Nephron 1976, 16:148-159.

3. Schubert C, Bates WD, Moosa MR: Acute tubulointerstitial nephritis related to antituberculous drug therapy. Clin Nephrol 2010, 73(6):413-419.

4. Izzedine H, Launay-Vacher V, Isnard-Bagnis C, Deray G: Drug-induced Fanconi's syndrome. Am J Kidney Dis 2003, 41(2):292-309.

5. Lino M, Binaut R, Noël LH, Patey N, Rustin P, Daniel L, Serpaggi J, Varaut $A$, Vanhille P, Knebelmann B, Grünfeld JP, Fakhouri F: Tubulointerstitial nephritis and Fanconi syndrome in primary biliary cirrhosis. Am J Kidney Dis 2005, 46(3):e41-e46.

6. Buysschaert M, Cosyns JP, Barreto L, Jadoul M: Pamidronate-induced tubulointerstitial nephritis with Fanconi syndrome in a patient with primary hyperparathyroidism. Nephrol Dial Transplant 2003, 18(4):826-829.

7. Hong YT, Fu LS, Chung LH, Hung SC, Huang YT, Chi CS: Fanconi's syndrome, interstitial fibrosis and renal failure by aristolochic acid in Chinese herbs. Pediatr Nephrol 2006, 21(4):577-579.

8. Neelakantappa K, Gallo GR, Lowenstein J: Ranitidine-associated interstitial nephritis and Fanconi syndrome. Am J Kidney Dis 1993, 22(2):333-336.

9. De Vriese AS, Robbrecht DL, Vanholder RC, Vogelaers DP, Lameire NH: Rifampicin-associated acute renal failure: pathophysiologic, immunologic, and clinical features. Am J Kidney Dis 1998, 31(1):108-115.

10. Racusen LC, Solez K: Nephrotoxic tubular and interstitial lesions: morphology and classification. Toxicol Pathol 1986, 14(1):45-57.

11. Gabow PA, Lacher JW, Neff TA: Tubulointerstitial and glomerular nephritis associated with rifampin. Report of a case. JAMA 1976, 235(23k0):2517-2518.
12. Praga M, González E: Acute interstitial nephritis. Kidney Int 2010, 77(11):956-961.

13. Rossert J: Drug-induced acute interstitial nephritis. Kidney Int 2001, 60(2):804-817.

14. Sugisaki T, Klassen J, Milgrom F, Andres GA, McCluskey RT: Immunopathologic study of an autoimmune tubular and interstitial renal disease in brown Norway rats. Lab Invest 1973, 28(6):658-671.

15. Neugarten J, Gallo GR, Baldwin DS: Rifampin-induced nephrotic syndrome and acute interstitial nephritis. Am J Nephrol 1983, 3(1):38-42.

16. Kohno K, Mizuta Y, Yoshida T, Watanabe H, Nishida H, Fukami K, lida S, Haramaki R, Wada Y, Tamai O, Tamaki K, Kato S, Morimatsu M, Okuda S: Minimal change nephrotic syndrome associated with rifampicin treatment. Nephrol Dial Transplant 2000, 15(7):1056-1059.

17. Yong $\mathrm{L}$, Killingsworth $M$ : Diffuse glomerulonephritis associated with rifampicin treatment for tuberculosis. Pathology 2002, 34(3):295-297.

18. Sebastian A, McSherry E, Morris RC Jr: Renal potassium wasting in renal tubular acidosis (RTA): Its occurrence in types 1 and 2 RTA despite sustained correction of systemic acidosis. J Clin Invest 1971, 50(3):667-678.

19. Sebastian A, McSherry E, Morris RC Jr: On the mechanism of renal potassium wasting in renal tubular acidosis associated with the Fanconi syndrome (type 2 RTA). J Clin Invest 1971, 50(1):231-243.

20. Rose BD, Post TW: Clinical Physiology of Acid-base and Electrolyte Disorder. 5th edition. New York: McGraw-Hill; 2001:612-627.

21. Feinfeld DA, Ansari N, Nuovo M, Hussain A, Mir R: Tubulointerstitial nephritis associated with minimal self reexposure to rifampin. $A m 」$ Kidney Dis 1999, 33(5):e3.

22. Yoshioka K, Satake N, Kasamatsu Y, Nakamura Y, Shikata N: Rapidly progressive glomerulonephritis due to rifampicin therapy. Nephron 2002, 90(1):116-118.

doi:10.1186/1471-2369-14-13

Cite this article as: Min et al:: Rifampin-associated tubulointersititial nephritis and Fanconi syndrome presenting as hypokalemic paralysis. BMC Nephrology 2013 14:13.

\section{Submit your next manuscript to BioMed Central and take full advantage of:}

- Convenient online submission

- Thorough peer review

- No space constraints or color figure charges

- Immediate publication on acceptance

- Inclusion in PubMed, CAS, Scopus and Google Scholar

- Research which is freely available for redistribution 